\title{
Provenances des semences de sorgho après une année de sécheresse au sud du Mali
}

Sonja Siart ${ }^{1}$

Eva Weltzien ${ }^{2}$

Moussa Kanouté ${ }^{2}$

Volker Hoffmann ${ }^{1}$

${ }^{1}$ Université de Hohenheim,

Département de communication

et vulgarisation agricole (430A)

70593 Stuttgart,

Allemagne

<sonja_siart@web.de>

<vohoff@uni-hohenheim.de>

2 International Crops Research Institute for the Semi-Arid Tropics (ICRISAT),

BP 320,

Bamako

Mali

<e.weltzien@icrisatml.org>

<kanoute_moussa@yahoo.fr>

\begin{abstract}
Résumé
Le sorgho (Sorghum bicolor) est l'aliment de base dans les régions du Mandé et de Dioila au sud du Mali. Les paysans y utilisent les semences issues de leur propre production. Afin de mieux comprendre les problèmes rencontrés par les paysans pour l'approvisionnement en semences suite à une année de sécheresse, des interviews individuelles ont été réalisées pendant la saison 2005. Les résultats montrent qu'après l'année de sécheresse, un pourcentage important de paysans a planté plus d'une seule variété de sorgho. D'après l'enquête, ils ont eu recours surtout à leurs propres semences, mais aussi à des semences en provenance de leur propre village et également d'autres villages, des projets et des marchés. Les transferts de semences ont été surtout basés sur des liens familiaux et de voisinage de sorte que l'accès aux semences ne dépendait pas de ressources financières. La raison principale pour la recherche de semences supplémentaires est surtout de pouvoir cultiver (20\%), ou tester, une nouvelle variété (7\%). Environ $10 \%$ des paysans n'avaient plus de stocks de semences. La décision concernant la source de semences et le choix de variété a été motivée et justifiée par la mauvaise production de l'année précédente. Cette baisse de production a conduit les paysans à augmenter la surface semée en sorgho pendant la saison 2005 et cela a causé un besoin accru de semences. Ce besoin a été satisfait par des semences de diverses sources locales qui ont permis l'introduction d'innovations variétales. Les paysans ont une flexibilité de réponse aux changements des conditions climatiques par la diversification de la gamme des variétés de sorgho et par l'augmentation de la surface cultivée. Le réseau social d'échanges de semences existant assure cette flexibilité et garantit la sécurité semencière. En conclusion, l'augmentation de la diversité variétale en collaboration avec les paysans devrait avoir des effets bénéfiques sur la sécurité alimentaire.
\end{abstract}

Mots clés : disponibilité alimentaire ; Mali ; pénurie alimentaire ; ressource génétique ; système semencier.

Thèmes : productions végétales ; ressources naturelles et environnement.

\section{Abstract \\ Farmers' sorghum seed sources after a drought year in southern Mali}

Sorghum (Sorghum bicolor) is the staple crop in the project regions Mandé and Dioila. Farmers in Mali use home-produced seed for sowing sorghum. In case of problems, like a drop in yield, farmers resort to using seed from other sources. To better understand what problems farmers in southern Mali may have with seed supply, individual interviews were conducted during the 2005 season, which followed a drought year. Results show that following the drought year a major percentage of farmers planted more than one sorghum variety. Farmers mentioned using other seed sources than their own production more frequently, especially from their own village, but also from other villages, projects and markets. Relations between the seed donors and seed recipients were mostly family ties and neighborhood relationships and thus seed access did not depend on financial resources. Reasons for looking for seeds from outside the household were mostly the interest in cultivating (20\%) or in testing (7\%) a new variety. About $10 \%$ of farmers mentioned that they had run out of seeds. Farmers had extended the area sown with sorghum due to the food shortages after the previous season. Thus, their decisions regarding seed source and variety choice were driven by their concern about the low cereal harvest the preceding year. Farmers did not face a major problem in seed availability and access within the local seed system after the drought year. The need for 
additional seeds could be satisfied with seeds from divers sources in the local system, providing an opportunity to introduce varietal innovations in the household portfolio. The fact that different farmers in a social seed network cultivate a range of different varieties contributes significantly to seed security and to farmers' flexibility to respond to changing climatic conditions and stresses. The improvement of variety diversity in cooperation with farmers can contribute to enhanced food security.

Key words: food shortages; food supply; genetic ressources; Mali; seed system.

Subjects: natural resources and environment; vegetal productions.

C ette étude s'intègre dans le cadre du projet de recherche agricole "Amélioration de l'accès à la diversité génétique du sorgho à travers la sélection participative " mis en oeuvre par l'International Crops Research Institute for the Semi-Arid Tropics (ICRISAT) Mali en collaboration avec des partenaires maliens et allemands et financé par le Bundesministerium für Wirtschaftliche Zusammenarbeit/Gesellschaft für Technische Zusammenarbeit (BMZ/GTZ)). Le sorgho [Sorgho bicolor (L.) Moench] est l'aliment de base des régions du projet, le Mandé et la région Dioila au sud du Mali. La pluviométrie dans cette zone varie entre 700 et $1000 \mathrm{~mm}$. La saison des pluies 2003 a été bonne pour la production du sorgho avec des pluies abondantes jusqu'en octobre. Une analyse détaillée du système semencier menée en 2004 (Siart, 2008 à paraître) a montré que la plupart des paysans n'ont pas rencontré de problème d'approvisionnement en semence, car $90 \%$ des unités de production agricole (UPA) ont eu un stock de semences satisfaisant. En 2004, les pluies ont été faibles et ont cessé en septembre, un mois plus tôt que d'habitude. Par conséquent, les rendements de sorgho ont été faibles et les paysans ont dû faire face à une récolte déficitaire. Au niveau national, la production céréalière a baissé en raison de la sécheresse et des attaques de criquets pèlerins au nord du pays ; elle a été de 2,845 millions de tonnes en 20042005 contre 3,409 millions de tonnes en 2003-2004 (Sidibé, 2006).

Sachant que les paysans utilisent une partie de leur production comme semences pour la saison suivante, une seconde enquête a été réalisée pendant la saison 2005 afin de mesurer l'influence d'une mauvaise saison des pluies sur la disponibilité en semences.

En utilisant une approche de recherche sociale empirique, des interviews individuelles basées sur un questionnaire stan- dardisé avec des questions ouvertes ont été conduites dans 16 villages. Une forme standardisée a été choisie en raison du nombre élevé d'échantillons ( $N=287$ en 16 villages) et pour permettre une comparaison avec les données d'analyse du système semencier obtenues en 2004 $(\mathrm{N}=451$ en 25 villages). Les questions ouvertes permettent aux interviewés de donner des réponses non influencées, avec leurs propres mots. Les interviewés étaient les chefs de familles. Ils sont responsables de la gestion des semences de sorgho dans la famille élargie et ils ont une profonde connaissance des variétés de sorgho. Dans chaque village, 15 à 20 chefs de familles ont été sélectionnés au hasard. Afin de mieux illustrer certains aspects, les résultats de l'enquête en 2004 ont été utilisés.

\section{Le système semencier}

L'enquête a porté sur les composantes du système semencier telle que la diversité variétale, la disponibilité et l'accessibilité de semences. L'évaluation de ces paramètres a aidé à identifier les forces et les faiblesses du système semencier local.

\section{Diversité variétale}

La diversité variétale du sorgho est généralement élevée dans les régions du projet. Au niveau des villages, 5 à 14 variétés différentes ont été identifiées. Les UPA ont cultivé entre 1 et 5 variétés et quelques UPA n'ont pas cultivé de sorgho. Les variétés ont différents noms locaux et ont été décrites par les paysans comme possédant des caractéristiques différentes. Quatre-vingt-quatorze pour cent des variétés mentionnées appartiennent à l'espèce Guinea de sorgho, qui est utili- sée pour préparer le tô ${ }^{1}$, l'aliment de base de la région. Les variétés locales améliorées issues de la recherche agricole sont rarement cultivées et sont surtout accessibles au travers des projets et services de vulgarisations.

Des différences entre les deux régions et entre les deux années ont été observées. La diversité variétale au niveau des UPA a augmenté après l'année de sécheresse dans les deux régions (figure 1). En 2005 , suite à l'année de sécheresse, un pourcentage élevé d'UPA dans la région de Dioila (69\%) et dans le Mandé $(62 \%)$ a cultivé plus d'une variété de sorgho. Ces résultats diffèrent de l'enquête de 2004, après une année de bonne production de sorgho, où 56 et $57 \%$ d'UPA dans la région de Dioilla et dans le Mandé avaient cultivé plus d'une variété.

La diversité variétale au niveau des UPA a donc augmenté après l'année de sécheresse de manière plus importante dans la région de Dioila $(+13 \%)$ que dans le Mandé (+ $5 \%$ ). Le nombre des UPA ne cultivant pas de sorgho a augmenté de $4 \%$ dans le Mandé et a diminué de $2 \%$ dans la région de Dioïla entre 2004 et 2005 .

En 2005, la raison principale invoquée pour chercher des semences supplémentaires était de pouvoir cultiver une nouvelle variété ( $20 \%$ du total des UPA). Une autre raison était la possibilité de pouvoir tester une nouvelle variété (7\%). Enfin, environ $10 \%$ de paysans ont dit avoir perdu leurs semences.

Dans la région de Dioïla, les paysans ont mentionné qu'ils ont étendu la surface semée en sorgho par rapport à la saison précédente. L'extension des surfaces cultivées en sorgho est l'une des stratégies pour surmonter une production céréalière insuffisante, avec comme consé-

\footnotetext{
${ }^{1}$ tô : bouillie traditionnelle préparée quotidiennement en Afrique de l'Ouest à partir d'une farine de sorgho, de mil ou de maïs.
} 


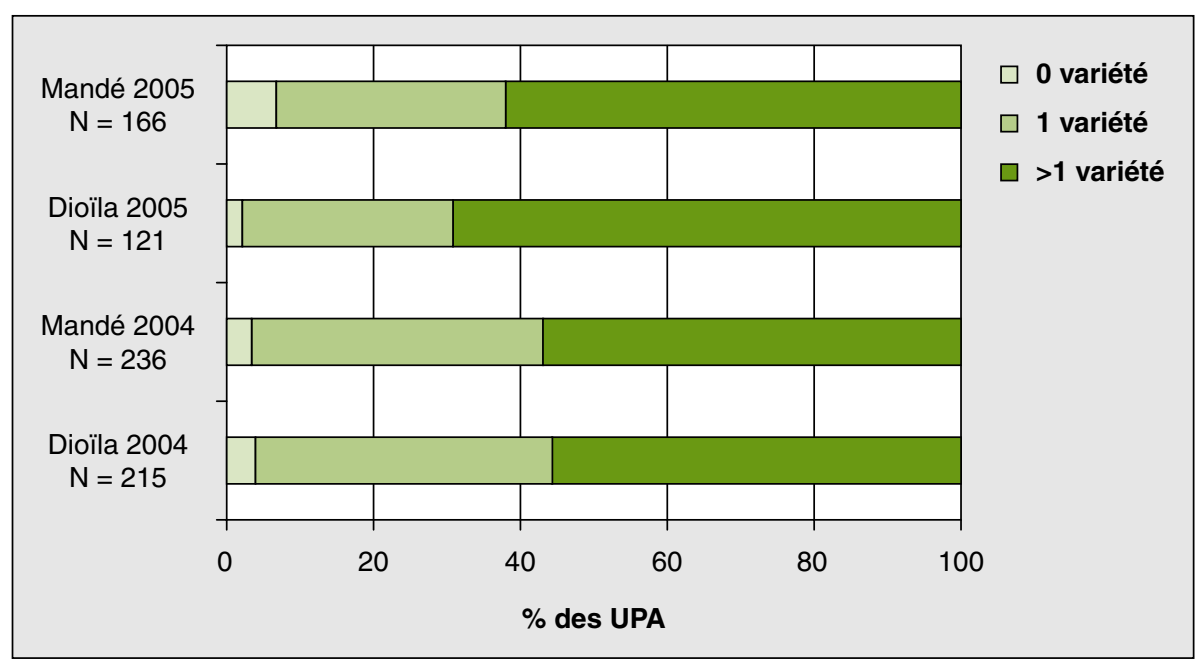

Figure 1. Diversité variétale au niveau des unités de production agricole (UPA) dans les régions de Dioïla et du Mandé en 2004 et 2005.

Figure 1. Varietal diversity at household level in Dioïla and Mandé in 2004 and 2005.

quence une augmentation du besoin en semences. Dans ce cas, les paysans sont parfois amenés à opter pour des variétés locales différentes de leurs variétés habituelles pour satisfaire leur besoin en semences supplémentaires.

Suite à la sécheresse, les familles gèrent donc une diversité variétale accrue, mais encore inférieure à celle trouvée au niveau du village. L'utilisation des nouvelles variétés avec des caractéristiques intéressantes pour les paysans, comme par exemple un cycle court, peut entrâner l'adoption de ces variétés au niveau de l'UPA. Ainsi, les innovations peuvent être introduites dans l'UPA en réponse aux situations précaires et aux variations climatiques.

\section{Disponibilité et accessibilité de semences}

L'augmentation de la diversité variétale au sein des UPA a résulté de la diversification de l'approvisionnement en semences pour la saison 2005. Le nombre des UPA capables de sauvegarder leurs propres semences a été de $90 \%$ en 2004 contre $83 \%$ en 2005 . Ainsi, en 2005, les paysans ont eu davantage besoin de semences supplémentaires. Les sources pour ces semences se trouvaient surtout au niveau de leur propre village (30\% du nombre total des UPA), mais aussi au niveau d'autres villages $(9 \%)$, des projets $(4 \%)$ et des marchés (3\%).

Les relations sociales impliquées dans les transferts de semences entre les paysans peuvent être regroupées en trois catégo- ries. Les semences sont recherchées auprès des membres de famille, les voisins et amis, et les personnes sans relation sociale forte tels les agents de projet, des services de vulgarisation ou les commerçants. Néanmoins, une différentiation exacte entre ces catégories n'est pas possible : par exemple, les voisins peuvent être également des parents. Les résultats
Figure 2. Seed sources at household level in 2005. montrent que les transferts des semences dans les deux régions se font surtout entre les membres de la famille (figure 2). Les deux catégories "membres de la famille " et "voisins et amis " ont été les plus importantes dans la région de Dioila. Dans le Mandé, la catégorie "voisins et amis " a été aussi importante que la catégorie "personnes sans relations sociales "; dans la dernière catégorie, les paysans ont placé surtout des services de vulgarisation et des projets, mais pas de commerçants. Ainsi, les paysans ont rarement mentionné le marché comme source de semence.

En 2005, les paysans ont obtenu les semences surtout par l'échange (27\%), le don $(23 \%)$ et l'achat (7\%). Bien que l'année 2004 ait été une année de sécheresse, certains producteurs de la communauté ont été capables de donner des semences ou au moins des grains stockés pour la consommation des variétés locales à d'autres paysans.

Aucun paysan n'a exprimé l'impossibilité de trouver des semences au niveau local. Comme les dons et les échanges sont les voies les plus importantes de transfert de semences, l'accès aux semences ne dépend pas directement des ressources financières, mais plutôt des relations sociales.

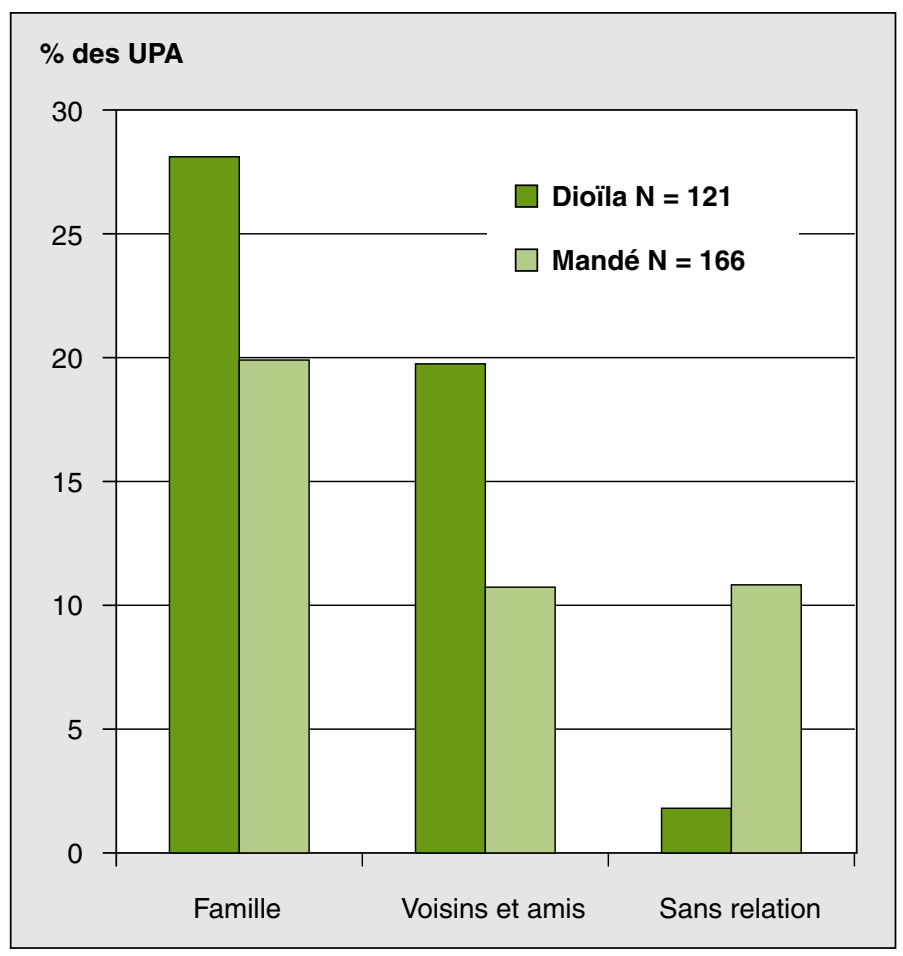

Figure 2. Sources de semences au niveau des unités de production agricole (UPA) en 2005. 


\section{Conclusion}

L'étude a montré que les paysans ont pu se procurer des semences au niveau local même après une année de sécheresse en cas de besoin de nouvelles variétés ou de semences additionnelles pour étendre la surface de sorgho. La majorité des paysans a sauvegardé ses propres semences et a accédé à d'autres variétés adaptées à l'extérieur des UPA. On peut donc conclure que le système semencier local répond aux exigences de sécurité semencière, définies par Sperling et Cooper (2003): disponibilité universelle de semences, accès des paysans aux semences et caractéristiques appropriées des semences.

Les choix de la source de semences et de la variété ont été déterminés par le faible niveau de la production de l'année précédente. La diversité variétale a augmenté au niveau des UPA en raison de la diversification des sources de semences et de l'extension des surfaces de sorgho, entrầnant un besoin accru de semences. L'objectif principal des paysans a été de réduire le risque d'un autre déficit de production.

Le fait que des paysans divers cultivent une gamme de différentes variétés dans un réseau social de semences contribue de façon significative à la sécurité semencière et à la capacité des paysans à répondre aux situations précaires et aux conditions climatiques variables. Les paysans minimisent les risques climatiques en utilisant des variétés fleurissant à différentes dates. Par conséquent, l'augmentation de la diversité variétale au niveau des UPA devrait avoir des effets positifs sur la sécurité alimentaire et sur la diversité génétique. En effet, si les conditions de culture et les préférences des paysans sont respectées, l'utilisation de ces variétés différentes contribue à leur conservation in situ. Ces résultats confirment l'importance de disposer d'une gamme de variétés différentes basées sur le germplasme local, incluant des variétés améliorées de cycles différents et au rendement élevé. Ces variétés doivent être gérées au niveau de la communauté et être mises à disposition par l'échange entre paysans.

\section{Références}

Siart S. Strengthening local seed systems options for enhancing diffusion of varietal options for enhancing diffusion of varietal
diversity of sorghum in southern Mali. Weikersheim (Germany): Margraf Verlag, 2008 (à paraître).

Sidibé O. L'impact de l'intégration sous régionale sur l'accès à l'alimentation des populations vulnérables au Mali. Deuxième Forum mondial des droits de I'homme, Cité des congrès de Nantes, 10-13 juillet 2006. http ://www.forum-droitsdelhomme.org/ml/ images/content/communication_ousmane sidibe.pdf.

Sperling L, Cooper D. Understanding seed systems and strengthening seed security. Background Paper prepared for Workshop on effecground Paper prepared for Workshop on effective and sustainable seed relief : A stakeholde workshop, Rome, 26-28 May, 2003. Rome :
Food and Agriculture Organization of the United Nations (FAO), 2003 